\title{
Enzymatic catalysis and transfers in solution. I. Theory and computations, a unified view
}

\author{
R. A. Marcus ${ }^{\text {a) }}$ \\ Noyes Laboratory of Chemical Physics, MC 127-72, California Institute of Technology, Pasadena, \\ California 91125-0072
}

(Received 23 August 2006; accepted 2 October 2006; published online 15 November 2006)

\begin{abstract}
The transfer of hydride, proton, or $\mathrm{H}$ atom between substrate and cofactor in enzymes has been extensively studied for many systems, both experimentally and computationally. A simple equation for the reaction rate, an analog of an equation obtained earlier for electron transfer rates, is obtained, but now containing an approximate analytic expression for the bond rupture-bond forming feature of these $\mathrm{H}$ transfers. A "symmetrization," of the potential energy surfaces is again introduced [R. A. Marcus, J. Chem. Phys. 43, 679 (1965); J. Phys. Chem. 72, 891 (1968)], together with Gaussian fluctuations of the remaining coordinates of the enzyme and solution needed for reaching the transition state. Combining the two expressions for the changes in the difference of the two bond lengths of the substrate-cofactor subsystem and in the fluctuation coordinates of the protein leading to the transition state, an expression is obtained for the free energy barrier. To this end a two-dimensional reaction space $(m, n)$ is used that contains the relative coordinates of the $\mathrm{H}$ in the reactants, the heavy atoms to which it is bonded, and the protein/solution reorganization coordinate, all leading to the transition state. The resulting expression may serve to characterize in terms of specific parameters (two "reorganization" terms, thermodynamics, and work terms), experimental and computational data for different enzymes, and different cofactor-substrate systems. A related characterization was used for electron transfers. To isolate these factors from nuclear tunneling, when the H-tunneling effect is large, use of deuterium and tritium transfers is of course helpful, although tunneling has frequently and understandably dominated the discussions. A functional form is suggested for the dependence of the deuterium kinetic isotope effect (KIE) on $\Delta G^{\circ}$ and a different form for the ${ }^{13} \mathrm{C}$ KIE. Pressure effects on deuterium and ${ }^{13} \mathrm{C}$ KIEs are also discussed. Although formulated for a one-step transfer of a light particle in an enzyme, the results would also apply to single-step transfers of other atoms and groups in enzymes and in solution.
\end{abstract}

(c) 2006 American Institute of Physics. [DOI: 10.1063/1.2372496]

\section{INTRODUCTION}

Enzymatic catalysis of hydride, proton, and $\mathrm{H}$ atom transfer has been the subject of many developments, past and recent, experimental and theoretical [e.g., articles in Philos. Trans. R. Soc. London, Ser. B. 361 (2006) and Chem. Rev. 106 (2006)]. In the theoretical studies there are extensive computations, based frequently on known enzyme structures, e.g., for recent reviews. ${ }^{1,2}$ The time scale for enzymatic $\mathrm{H}$ transfers is usually of the order of milliseconds, whereas current numerical computations utilize classical mechanical trajectories of the atoms lasting about $10 \mathrm{~ns}$. So commonly some other form of theory, typically transition state theory, is used to calculate the reaction rate. The discussion in this article is intended to apply both to reactions in enzymes and to reactions in solution when, in the latter case, one converts the usual second-order rate constant to a first-order one for the "collision complex." While couched in terms of a single step $\mathrm{H}$ transfer, the formalism is intended to apply also to the single-step transfer of other atoms and groups, and can be extended to multiple steps.

Among the factors contributing to the reaction rate in a

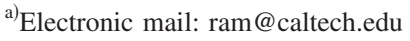

bound substrate-cofactor enzyme complex are (1) the work required for the reactants in the bound complex to approach each other closely enough to optimize the H-transfer rate, (2) an additional energy barrier accompanying the bond rupture and bond formation in the substrate-cofactor complex, and (3) fluctuations in the structure of the enzyme and in solution (in electron transfers called "reorganization") so as to reach the transition state (TS) for the reaction. The electronic polarization at each point in the enzyme influences the energy barrier and is (or should be) treated as a "fast" variable, e.g., as in Ref. 3 for electron transfer. In addition, there is frequently (4) a nuclear tunneling of the light atom, $\mathrm{H}^{\circ}, \mathrm{H}^{-}$, or $\mathrm{H}^{+}$, so enhancing a H/D kinetic isotope effect (KIE) on the rate of the transfer. This KIE typically varies between 3 and 100 , depending on the system, and is superimposed on a much larger variation in the ratio, enzymatic rate/rate in solution, that may vary from $\sim 10^{6}$ to $10^{17}$.

The question we address in the present paper is whether an equation can be designed for these ion and atom transfer reactions that is an analog of a widely used expression given in Ref. 4 and references cited therein for the rates of electron transfer reactions. In the latter field, terms such as "outer reorganization energy" $\lambda_{o}$, inner reorganization energy $\lambda_{i}$, 
standard free energy of reaction $\Delta G^{\circ}$, work terms $w^{r}$ and $w^{p}$, and electronic coupling matrix element $V$ are standard terminologies. These properties are deduced from the experimental data, or from calculations, or from both. We explore whether some analogous framework can be formulated for $\mathrm{H}^{*}, \mathrm{H}^{+}$, and $\mathrm{H}^{-}$transfers. One result is given in Eqs. (10), (18), and (22). Postulated functional forms for the free energy dependence of deuterium and ${ }^{13} \mathrm{C}$ kinetic isotope effects are given in Eqs. (36)-(39).

Two of the principal approaches used in numerical computations of rates of the enzymatic transfer of a $\mathrm{H}^{*}, \mathrm{H}^{+}$, or $\mathrm{H}^{-}$ between substrate and cofactor have the following quite different origins, with subsequent refinements.

(1) In a reaction between a donor $\mathrm{DH}$ and an acceptor $\mathrm{A}$, $\mathrm{DH}+\mathrm{A} \rightarrow \mathrm{D}+\mathrm{HA}$, where $\mathrm{H}$ can be $\mathrm{H} \mathrm{H}^{\cdot}, \mathrm{H}^{+}$, or $\mathrm{H}^{-}$, the difference of bond lengths of the dissociating (DH) bond and the newly forming (HA) bond is used as a reaction coordinate, e.g., by Gao et al. ${ }^{1}$ The free energy of formation of the transition state from the reactants is calculated and, with it, the reaction rate. A nuclear tunneling effect on the rate is added.

(2) Instead, the vertical energy difference $\Delta E$ of the two lowest diabatic empirical valence bond (EVB) structures (e.g., DH, A and D, HA) is used as a reaction coordinate, e.g., by Aqvist and Warshel ${ }^{5}$ and Billeter et $a l^{6}{ }^{6}$ The free energy of an ensemble of configurations for each of the EVB structures is calculated as a function of $\Delta E$. When $\Delta E=0$ the system is taken to be in the transition state. For each $\Delta E$ this ensemble lies on an $N-1$ dimensional hypersurface in an $N$-dimensional coordinate space. (Because of momentum conservation, and an application of an approximate Franck-Condon principle to $\mathrm{H}$ transfers, the same result is obtained in phase space.) $\Delta E$ was used earlier as a reaction coordinate for electron transfer (ET) reactions by Marcus ${ }^{7}$ and by King and Warshel. ${ }^{8}$ Frequently the $V$ defined below is included in determining the energy of the TS in the $\Delta E$-based ET calculation, but the difference in the position of TS is usually small. ${ }^{5}$

If $V$ denotes the matrix element coupling the two lowest EVB diabatic electronic states, the energy of the TS is reduced in magnitude by the "resonance energy" $V$ of the two structures, as in Fig. 1. When this EVB approach was used" to treat experiments in enzymes, the value of $V$ was adjusted so that the free energy barrier $\Delta G^{*}$ for the corresponding reaction rate in solution agreed with the experimental value. This result for $V$ was then used to calculate the $\Delta G^{\dagger}$ for the rate of the enzyme catalyzed reaction. Tunneling corrections were again introduced.

In the EVB approach a reorganization of the system occurs. There are fluctuations in the positions of all the atoms in the protein so as to reach the transition state, a state in which the two lowest EVB states have the same energy. On the other hand, in the bond length difference approach, there are again fluctuations in the enzyme structure to reach the TS, but a functional form for any such reorganization is not explicitly singled out. Yet another approach, based on the centroid approximation, ${ }^{10,11}$ has also been used. ${ }^{12}$

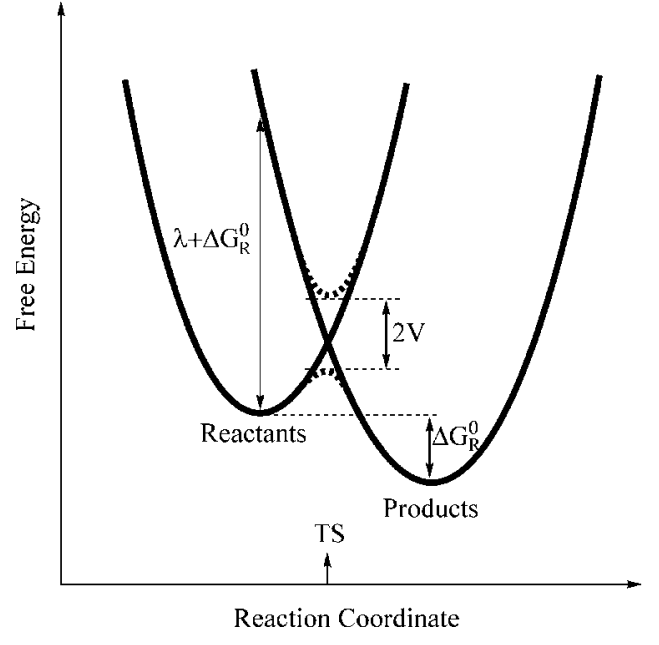

FIG. 1. Plot of free energy vs reaction coordinate for the reactants and for the products. The heavy solid lines indicate the curves for the diabatic states, the adiabatic curves being indicated by the dotted curves. $\Delta G_{R}^{\circ}, \lambda+\Delta G_{R}^{\circ}$ and $V$ mentioned in the text are indicated.

We explore the possibility of unifying the two principal approaches, with the goal of obtaining an equation for the $\mathrm{H}$ transfer rate constant that contains concepts used in both, and permits the use of an $a b$ initio potential energy surface rather than an EVB-type of approximation, if it is available. On the other hand, EVB yielded very useful pioneering results. A merit of the EVB approach is that in the numerical calculations it showed a simple quadratic behavior for the free energy barrier $\Delta G^{\dagger}$ due to the fluctuations of the protein leading to the TS (Gaussian fluctuations). While some simple quadratic behavior of the protein might be occurring in the bond length difference formalism, it was not explicitly identified there. At present, it is not known how much of the quadratic behavior is due to the two-state EVB model, which has two distinct charge distributions.

In exploring a "synthesis" of the two approaches, we first review some relevant aspects of ET theory. Earlier approximate models of $\mathrm{H}$ transfers in solution or in enzymes include those of Albery, ${ }^{13}$ Borgis and Hynes, ${ }^{14}$ Antoniou and Schwartz, ${ }^{15}$ Kiefer and Hynes ${ }^{16,17}$ and Kuznetsov and Ulstrup ${ }^{18}$ in addition to the works already cited.

The present approach is intended to be complementary to the many extensive and important computations on $\mathrm{H}$ and other transfer reactions in these systems. The article is organized as follows: an overview of electron transfer theory is given in Sec. II, and an equation for $\mathrm{H}^{+}, \mathrm{H}^{-}$, and $\mathrm{H}^{\bullet}$ transfers is obtained in Sec. III, key equations being Eqs. (10), (18), and (22). The assumptions are discussed there. The absence of charge transfer spectra is discussed in Sec. IV, raising the question of possible observation at high pressures. A second $\Delta E$ reaction coordinate, commonly used in the EVB-based solutions, is also discussed there. Nuclear tunneling is treated in Sec. V, where approximate functional forms are suggested for the dependence of the deuterium and ${ }^{13} \mathrm{C}$ kinetic isotope effects on the driving force [(Eqs. (36)-(39)]. Concluding remarks are given in Sec. VI.

\section{REVIEW OF ELECTRON TRANSFER THEORY}

We recall an equation and concepts used in ET theory, and then modify them so as to treat $\mathrm{H}^{*}, \mathrm{H}^{+}$, or $\mathrm{H}^{-}$transfers, 
transfers that also contain simultaneous bond breaking and bond forming. The equation for a "unimolecular" rate constant $k_{\mathrm{et}}$ of an electron transfer reaction occurring in a reaction complex between two molecular species is given by Marcus ${ }^{19}$ and Marcus and Sutin $^{4}$ and references cited therein:

$$
k_{\mathrm{et}}=A e^{-\Delta G^{*} / k T},
$$

where

$$
\Delta G^{*}=w^{r}+\left(\lambda+\Delta G^{\circ}(R)\right)^{2} / 4 \lambda
$$

and

$$
\Delta G^{\circ}(R)=\Delta G^{\circ}+w^{p}(R)-w^{r}(R) .
$$

The preexponential factor $A$ depends on the electronic coupling $V$ of the two reactants. When the reactants are weakly coupled electronically in the transition state the reaction is nonadiabatic and $A$ is proportional to $|V|^{2}$. When the electronic coupling $V$ is instead strong in the TS region (adiabatic reaction) $A$ is of the order of a vibration frequency. $w^{r}(R)$ is the work done (electrostatic work, for example) to bring the two reactants from their equilibrium positions in the complex to a separation distance $R$ that facilitates the electron transfer, and $w^{p}(R)$ is the corresponding quantity for the products, $\lambda$ is the "reorganization energy," $\Delta G^{\circ}$ the standard free energy of the reaction in the bound complex, and $\Delta G^{\circ}(R)$ is defined by Eq. (3). The $w^{r}(R)$ and $w^{p}(R)$ denote the part of the free energy barrier that is not overcome by a favorable $\Delta G^{\circ}(R)$.

The $\lambda$ and $\Delta G^{\circ}(R)$ are indicated in Fig. 1 for electron transfers in terms of the customary free energy parabolas for reactants and products, symmetrized as in Ref. 19. An approximate vibrational and dielectric continuum-based equation for $\lambda$ illustrates features of the reorganization, taken from Ref. 7,

$$
\lambda=(\Delta e)^{2}\left(\frac{1}{D_{\mathrm{op}}}-\frac{1}{D_{s}}\right)\left(\frac{1}{2 a_{1}}+\frac{1}{2 a_{2}}-\frac{1}{R}\right)+\frac{1}{2} \sum_{i} k_{i}\left(\Delta q_{i}\right)^{2},
$$

where $\Delta e$ is the charge transferred. In a weak-overlap reaction usually one electron per successive electron transfer step is preferred energetically over a single-step multiple electron transfer, i.e., usually $\Delta e=e$, the electronic charge. $D_{\mathrm{op}}$ and $D_{s}$ are the optical and static dielectric constants of the medium surrounding the pair of reactants. In the particular form for $\lambda$ given in Eq. (4) the reactants are treated as spheres of radii $a_{1}$ and $a_{2}$ separated by a center-to-center distance $R ; k_{i}$ is the symmetrized "force constant" of the $i$ th normal mode vibration of the reactants, symmetrized as in Ref. 19, and $\Delta q_{i}$ is difference in equilibrium positions of the $i$ th normal mode in the products and in the reactants. The $\left(1 / D_{\mathrm{op}}-1 / D_{s}\right)$ in Eq. (4) arises because of the difficulty of the slow polarization dielectric response of the solvent to satisfy at the same time the two very different spatial charge distributions in the TS, that of the reactants and that of the products. ${ }^{20}$

In a refinement of these equations, $\left(1 / D_{\mathrm{op}}-1 / D_{s}\right)$ is replaced by an expression that contains the dielectric dispersion of the medium, introducing thereby a dynamical behavior of the surrounding solvent into more than just one fast
$\left(D_{\mathrm{op}}\right)$ and one slow $\left(D_{s}\right)$ dielectric response, e.g., in Ref. 21. Other geometries have also been treated, such as an ellipsoid instead of a pair of spheres. Molecular based statistical mechanical expressions have also been used instead of a continuum expression, e.g., in Refs. 7 and 22. Quantum expressions for the high frequency normal mode vibrations of the reactants have also been introduced, e.g, by Kestner et $a l^{23}$ The latter can be particularly important in the "inverted region" (the region where $\lambda<-\Delta G_{R}^{\circ}$ ). Molecular-based numerical computations of the reorganization energy $\lambda$ and of $\Delta G^{\circ}$ have been made, e.g., by Warshel et al. ${ }^{24}$ Kuharski et al. ${ }^{25}$ and King and Warshel. ${ }^{8}$

The solid curves in Fig. 1 illustrate a key feature of the transition state for weak-overlap electron transfer reactions: A consequence of the Franck-Condon principle is that in the TS there is no change of atomic positions and momenta during the ET. Hence, energy is conserved during the electron transfer in the TS, namely, the latter occurs at coordinates defining the intersection of reactants' and products' potential energy surfaces in many-dimensional coordinate space. The latter was then expressed in terms of free energy plots, such as in Fig. 1, using statistical mechanics, as Refs. 7 and 19. The electronic polarization of the environment is again a "fast variable," leading, in a continuum description, to the presence of the $D_{\text {op }}$ in Eq. (4) instead of the unity that would occur in the absence of a correlative shielding effect of the fast (electronic) polarization. The latter thus has a dramatic effect on the reaction rate.

\section{H TRANSFERS}

In considering the $\mathrm{H}^{+}, \mathrm{H}^{-}$, or $\mathrm{H}^{*}$ transfer reactions we first neglect nuclear tunneling, and later consider tunneling in Sec. V. For a nonadiabatic electron transfer the quantum mechanical "splitting" $2 V$ of the two lowest adiabatic potential energy surfaces at the TS for the reacting species and their surroundings is very small, and so the "intersection" of the solid lines in Fig. 1 is really an avoided crossing with a small splitting $2 V$. However, for the $\mathrm{H}$ transfer systems, this $2 \mathrm{~V}$ in Fig. 1 is frequently large, perhaps $\sim 1 \mathrm{eV}$ instead of $\sim 0.02 \mathrm{eV}$, because chemical bonds are breaking and forming. So we can no longer invoke an energy equality to define the TS in Fig 1.

The DHA reacting pair is defined as consisting of the atoms directly or closely involved in the covalent bond breaking-bond forming reaction, frequently some 50 or so atoms. The totality of their coordinates is denoted by $\boldsymbol{q}_{\mathrm{DHA}}$. The remaining system, i.e., the rest of the substrate-cofactor complex, enzyme, and the surrounding solution, will be abbreviated as "protein." It is denoted throughout by "prot" and its coordinates by $\boldsymbol{q}_{\text {prot }}$. The division is similar to that used in QM/MM computations, quantum mechanics for part of DHA and molecular mechanics for the rest of the system.

To treat these adiabatic $\mathrm{H}$ transfers using ab initio or other high level quantum calculations for the DHA reacting pair, the vertical energy difference of the two lowest adiabatic electronic states of the system $\Delta E$ in the manydimensional coordinate space can still be a useful coordinate. The nuclear coordinates of the system are sampled, including 
those of the DHA reacting pair. At each value of $\Delta E$ there is a thermal ensemble of configurations in the manydimensional coordinate space and as in electron transfers it yields the free energy. This free energy is a function of $\Delta E$ for each of the two lowest adiabatic states. The free energy of the lowest adiabatic state reaches a maximum at the TS, and the vertical energy difference $\Delta E$ of the two lowest adiabatic states typically reaches a minimum there, instead of having the nearly zero value that it has in weak-overlap electron transfers and that it would have in any weak-overlap $\mathrm{H}$ transfer. We denote the $\Delta E$ of the two lowest adiabatic states by $\Delta E_{\text {total }}$. In a different $\Delta E$ coordinate only the protein coordinates are sampled, the coordinates of the DHA reacting pair being treated separately. We denote this $\Delta E$ by $\Delta E_{\text {prot}}$.

In EVB computations the free energy of the reacting pair was a quadratic function of $\Delta E_{\text {total }}{ }^{5}$ and also a quadratic function of $\Delta E_{\text {prot }}{ }^{26}$ Analogous results in these and other problems are understood analytically in terms of a cumulant expansion, e.g., Refs. 12, 22, and 24. Initially, we use $\Delta E_{\text {prot }}$ as a coordinate and later in Sec. IV extend the analysis to $\Delta E_{\text {total }}$.

The potential energy term $U$ in the Schrödinger equation for the electronic state of the reacting pair can be written as

$$
U=\sum_{i-j} \frac{e_{i} e_{j}}{r_{i j}}+\sum_{i} e_{i} \phi_{i}
$$

where the $e_{i}$ denote the charges of the electrons and the nuclei in the reactants, and $\phi_{i}$ is the potential acting on each $e_{i}$ due to all charges and dipoles in the enzyme outside the reacting pair; $\phi_{i}$ contains any interaction with the electronic polarization of the region outside the reacting pair.

Upon solving the Schrödinger equation for the reacting pair for the $U$ in Eq. (5), the energy of the entire system $E$ can be written as

$$
E=E_{\mathrm{DHA}}\left(\boldsymbol{q}_{\mathrm{DHA}}\right)+E_{\text {prot }}\left(\boldsymbol{q}_{\mathrm{prot}}, \boldsymbol{q}_{\mathrm{DHA}}\right),
$$

where $E_{\mathrm{DHA}}\left(\boldsymbol{q}_{\mathrm{DHA}}\right)$ is the part of $E$ that is the electronic energy of the 50 or so principal atoms in the substrate-cofactor complex. It varies along the reaction coordinate and is particularly sensitive to the difference in $\mathrm{DH}$ and HA distances. $E_{\text {prot }}\left(\boldsymbol{q}_{\text {prot }}, \boldsymbol{q}_{\text {DHA }}\right)$ is the sum of the interaction energy of the reacting pair (DH,A) with the protein and the interaction of the protein with itself.

The free energy $G$ associated with $\Delta E_{\text {prot }}$ for any given $\boldsymbol{q}_{\text {DHA }}$ can be written as

$$
\begin{aligned}
G\left(\boldsymbol{q}_{\mathrm{DHA}}, \Delta E_{\mathrm{prot}}\right) & =E_{\mathrm{DHA}}\left(\boldsymbol{q}_{\mathrm{DHA}}\right)-k T \ln \int e^{-\left(E-E_{\mathrm{DHA}}\right) / k T} d \Gamma \\
& \equiv E_{\mathrm{DHA}}\left(\boldsymbol{q}_{\mathrm{DHA}}\right)+G_{\text {prot }}\left(\boldsymbol{q}_{\mathrm{DHA}}, \Delta E_{\mathrm{prot}}\right),
\end{aligned}
$$

where $d \Gamma$ is the volume element in the many-dimensional coordinate space for $\boldsymbol{q}_{\text {prot }}$.

Prior to bond breaking-bond forming, there may be work $w^{r}$ needed to bring the reacting pair in the bound complex to some separation distance $R$ to facilitate the subsequent transfer of the H. This $w^{r}$ is the part of the free energy barrier not reduced by a favorable $\Delta E_{\mathrm{DHA}}^{\circ}$ nor by a favorable $\Delta G_{\text {prot }}^{\circ}$.

A bond energy-bond order (BEBO) method was introduced by Johnston ${ }^{27}$ for atom or group transfer reactions. In this method, sum of the DH and HA bond orders for the $\mathrm{H}$ is assumed to be a constant, unity, during the motion along the reaction coordinate. In the modified and symmetrized BEBO approximation given in Ref. 28 , we write $E_{\text {DHA }}\left(\boldsymbol{q}_{\text {DHA }}\right)$ as $E_{\mathrm{DHA}}(n, R)$, where there is now an averaging over all $\boldsymbol{q}_{\mathrm{DHA}}$ for the given $n$ and $R$. We have, ${ }^{28}$ relative to the value of $E_{\mathrm{DHA}}(n, R)$ at $n=0$,

$$
\begin{aligned}
E_{\mathrm{DHA}}(n, R)= & -\frac{\lambda_{i}}{4 \ln 2}[n \ln n+(1-n) \ln (1-n)] \\
& +n \Delta E_{\mathrm{DHA}}^{\circ}(R),
\end{aligned}
$$

where $n$ is the bond order of the newly forming H-A chemical bond and $\Delta E_{\mathrm{DHA}}^{\circ}(R)$ is the electronic energy of reaction of the bound substrate-cofactor complex at the given $R$. One sees in Eq. (8) that at $n=1, E_{\mathrm{DA}}=\Delta E_{\mathrm{DHA}}^{\circ}(R)$. In the symmetrization leading to Eq. (8) a term asymmetric in the two bond energies, a term that vanishes at $n=0,1 / 2$ and 1 , was presumed to be small and was neglected. $^{28}$

One can explore fitting Eq. (8) to the computations of $E_{\mathrm{DHA}}(n, R)$. Upon finding the local maximum in Eq. (8), namely, the $n$ that satisfies

$$
\left(\lambda_{i} / 4 \ln 2\right) \ln [n /(1-n)]=\Delta E_{\mathrm{DHA}}^{\circ}(R),
$$

one obtains an expression for this contribution $E_{\mathrm{DHA}}(n, R)$ to the energy barrier for reaction. In the absence of any correlation between $n$ and a protein/solvent reorganization coordinate $m$, the resulting energy barrier $\Delta E_{\mathrm{DHA}}^{*}(R)$ is given by ${ }^{28}$

$$
\Delta E_{\mathrm{DHA}}^{*}(R)=\frac{\lambda_{i}}{4}+\frac{\Delta E_{\mathrm{DHA}}^{\circ}(R)}{2}+\frac{\Delta E_{\mathrm{DHA}}^{\circ}(R)}{2 y} \ln \cosh y,
$$

where $\lambda_{i} / 4$ in Eq. (10) is the mean of the properties of the two bonds, given in Eq. (8c) of Ref. 28, and $y$ is given by

$$
y=\left(2 \Delta E_{\mathrm{DHA}}^{\circ}(R) \ln 2\right) / \lambda_{i} \text {. }
$$

The $\lambda_{i} / 4$ in Eq. (10) can be obtained by extrapolating or interpolating this contribution to the energy barrier $\Delta E_{\mathrm{DHA}}^{*}(R)$, experimentally or computationally, to its value at $\Delta E_{\mathrm{DHA}}^{\circ}(R)=0$.

We consider next the protein reorganization. The free energy well for the reactants is harmonic in the vicinity of latter's minimum and similarly for products' well. When $\mathrm{H}$ approaches midway between the heavy atoms $(n \rightarrow 1 / 2)$, the analog of the two very different charge distributions present in "weak overlap" electron transfers disappears. Accordingly there can be some deviation from the harmonic behavior, as in the dashed line in Fig. 1. In Fig. 1 a "rounding off" arose from a coupling term $V$. However, now in addition the rounding off is augmented by the merging of the two charge distributions, a merging that modifies the protein reorganization term when $n$ is in the vicinity of $1 / 2$.

For simplicity we nevertheless use the quadratic form to approximate the $G_{\text {prot }}(m)$ in Eq. (7) and use as its reaction coordinate the $m$ in ET theory. The physical significance of $m$ is described later. We then have 


$$
\Delta G_{\mathrm{prot}}(m)=m^{2} \lambda_{o} \quad\left(m<m_{c}\right)
$$

and for the reverse reaction, starting from the products $p$,

$$
\Delta G_{\mathrm{prot}}^{p}(m)=(1-m)^{2} \lambda_{o} \quad\left(m>m_{c}\right),
$$

where $\lambda_{o}$ is the reorganization energy of the protein and $m_{c}$ is the $m$ which satisfies the intersection condition

$$
\Delta G_{\text {prot }}(m)=\Delta G_{\text {prot }}^{p}(m)+\Delta G_{\text {prot }}^{\circ} .
$$

Thereby, $m_{c}$ satisfies

$$
(1-2 m) \lambda_{o}=-\Delta G_{\mathrm{prot}}^{\circ} .
$$

For symmetry, we replaced $m$ in Refs. 7, 19, and 20 by $-m$, so that $m$ in the "normal" region now lies in the interval $(0,1)$ instead of $(-1,0)$. From Eqs. (13) and (14) we have, for the lowest adiabatic state when $m>m_{c}$, instead of Eq. (13),

$$
\Delta G_{\mathrm{prot}}(m)=(1-m)^{2} \lambda_{o}+\Delta G_{\mathrm{prot}}^{\circ}\left(m>m_{c}\right) .
$$

The physical significance of $m$ is that it describes the state of the protein at that $m$. For example, if $\boldsymbol{P}_{\text {orient }}^{r}(\boldsymbol{r})$ denotes the orientation polarization for reactants'/protein system in an equilibrium state at any $\boldsymbol{r}$ and $\boldsymbol{P}_{\text {orient }}^{\boldsymbol{p}}(\boldsymbol{r})$ denotes that for the products in and equilibrium state, then $\boldsymbol{P}_{\text {orient }}^{(m)}(\boldsymbol{r})$, the orientation polarization in the protein at $\boldsymbol{r}$ for any state $m$, is given by

$$
\boldsymbol{P}_{\text {orient }}^{(m)}(\boldsymbol{r})=(1-m) \boldsymbol{P}_{\text {orient }}^{r}(\boldsymbol{r})+m P_{\text {orient }}^{p}(\boldsymbol{r}) .
$$

When the value of $m$ is that for the TS, $\boldsymbol{P}_{\text {orient }}^{(m)}(\boldsymbol{r})$ is the orientation polarization for the TS. When $\Delta G^{\circ}$ (prot) is zero, $\boldsymbol{P}_{\text {orient }}^{(m)}(\boldsymbol{r})$ in the TS is seen to be the arithmetic mean of its initial and final equilibrium values. In the TS, $P_{\text {orient }}^{(m)}(\boldsymbol{r})$ is a fluctuation from these values.

From Eqs. (15) and (16) one obtains an expression for the protein reorganization contribution to the free energy barrier to the reaction at the given $R$ :

$$
\Delta G_{\mathrm{prot}}^{*}=\left[\lambda_{o}+\Delta G_{\mathrm{prot}}^{\circ}\right]^{2} / 4 \lambda_{o},
$$

where

$$
\Delta G_{\mathrm{prot}}^{\circ}=\Delta G^{\circ}(R)-\Delta E_{\mathrm{DHA}}^{\circ}(R)
$$

and $\Delta G^{\circ}(R)$ is given by Eq. (3).

In an analysis of computational results it is also useful to have an expression for an energy difference coordinate $\Delta E_{\text {prot }}$ that describes the protein reorganization. On using the continuation of Eq. (16) to $m<m_{c}$ we have

$$
\Delta E_{\text {prot }}(m)=(1-2 m) \lambda_{o}+\Delta G_{\text {prot }}^{\circ} \quad\left(m<m_{c}\right)
$$

and analogously

$$
\Delta E_{\text {prot }}(m)=-(1-2 m) \lambda_{o}-\Delta G_{\text {prot }}^{\circ}\left(m>m_{c}\right) .
$$

These equations relating $\Delta E_{\text {prot }}(m)$ to $m$ neglect the rounding off mentioned earlier that occurs when $n$ is in the vicinity of $1 / 2$.

In the approximation leading to Eq. (18), the assumption of no rounding off, leads to this being no $(n, m)$ cross term. The free energy barrier then contains the sum of an $n$-dependent term, $m$-dependent term, and $w^{r}$. In the absence of nuclear tunneling, we then have

$$
k_{\text {rate }}=A e^{-\Delta G^{*} / k T}
$$

where

$$
\Delta G^{*}=w^{r}+\Delta E_{\mathrm{DHA}}^{*}(R)+\Delta G_{\mathrm{prot}}^{*}
$$

and the last two terms are given by Eqs. (10) and (18). We include in the $w^{r}\left(w^{p}\right)$ any reorientation or conformational change of the two reactants (products) and any change of zero-point energy of the $\mathrm{H}$ vibrations (stretching and two bending vibrations) to reach the TS from the reactants (products), in the bound complex.

Certain limiting forms of Eq. (22) are immediately obtained.

(a) When both $\Delta G_{\text {prot }}^{\circ}=0$ and $\Delta E_{\mathrm{DHA}}^{\circ}(R)=0$ the solutions of the equations for $n$ and $m$ are $n=1 / 2$ and $m=1 / 2$. Then

$\Delta G_{R}^{*}=w^{r}+\lambda / 4$,

where $\lambda=\lambda_{i}+\lambda_{o}$, an expression familiar from electron transfer theory, e.g., as in Ref. 19.

(b) When $\Delta E_{\mathrm{DHA}}^{\circ}(R) \rightarrow-\infty$ then the $\Delta E_{\mathrm{DHA}}^{*}(R)$ term vanishes but the $\Delta G_{\text {prot }}^{*}$ would show an inverted effect if $\left|\Delta G_{\text {prot }}^{\circ} / \lambda_{o}\right|>1$. However, this effect neglects the rounding off mentioned earlier. Further, the spacing of the protonic quantum states of the products is small compared with that of the electronic states of the products and there can be expected to be a smaller inverted effect when reaction into the excited protonic states of the product HA is included.

(c) When $\Delta E_{\mathrm{DHA}}^{\circ}(R)$ is small, Eq. (10) can be expanded to the terms quadratic in the $\Delta G^{\circ}$ and $\Delta E_{\mathrm{DHA}}^{\circ}(R)$, yielding

$$
\begin{aligned}
\Delta G^{*}= & w^{r}+\frac{\lambda_{o}+\lambda_{i}}{4}+\frac{\Delta G^{\circ}}{2}+\left(\frac{\Delta G_{\mathrm{prot}}^{\circ 2}}{4 \lambda_{o}}\right) \\
& +\frac{\left(\Delta E_{\mathrm{DHA}}^{\circ}(R)\right)^{2} \ln 2}{2 \lambda_{i}} \\
& +\cdots \quad\left(\Delta E_{\mathrm{DHA}}^{\circ} / \lambda_{i} \text { is small }\right) .
\end{aligned}
$$

The approximations leading to Eqs. (10), (18), and (22) are several fold: (i) Gaussian fluctuations for the protein, including its interaction with the substrate and cofactor; (ii) a symmetrized and simplified "BEBO" functional form for the DHA potential energy as a function of a bond order coordinate $n$ of the newly forming bond; and (iii) neglect of the $(n, m)$ cross term in the vicinity of $n=1 / 2$. Approximation (iii) occurred when the effect of collapse of the distinct charge distributions into one when $n=1 / 2$ was neglected. It will be recalled that it was the two distinct charge distributions that led to the $D_{\text {op }}$ term in Eq. (4). This effect of the merging of the two charge distributions in the vicinity of $n$ $=1 / 2$ does not appear to have been explored in existing high level quantum chemistry $\mathrm{H}^{+}, \mathrm{H}^{-}$transfer calculations, The change of charge distribution along the coordinate $n$ in the vicinity of $n=1 / 2$ was noted, in a different language, in Ref. 1. A test of approximation (iii) is discussed in Sec. V.

The physical idea of the BEBO approximation is seen in a symmetric exchange reaction, such as $\mathrm{CH}_{3}+\mathrm{CH}_{4} \rightarrow \mathrm{CH}_{4}$ $+\mathrm{CH}_{3}$ : The activation energy is roughly $10 \%$ of the $\mathrm{CH}$ bond 
dissociation energy. That is, the total bond order is roughly preserved at unity during the reaction. A definition of $n$ has been given in terms of the deviation $r-r_{e}$ from an equilibrium bond length $r_{e}$, as in Ref. 29 and as described in Ref. 30 ,

$$
r-r_{e}=-\beta \ln n+\text { const, }
$$

where $\beta$ is a constant. However, when applied to computations for these reactions, this equation can lead to a serious error in the vicinity of $n=0$ or $n=1$ : When $n$ is near unity for one bond it is near zero for the other, for which strictly speaking only distances of $r=\infty$ apply. This problem leads to minor error in $\Delta E_{\mathrm{DHA}}(n, R)$ in Eq. (8), since even though $\ln n \rightarrow-\infty$ when $n \rightarrow 0, n \ln n \rightarrow 0$. Instead of relating $r-r_{e}$ to $n$, one can avoid the $r=\infty$ singularity by expanding about $n=1 / 2$, the most common region for the TS:

$$
r-r_{1 / 2}=\beta \ln 2 n
$$

so that $r=r_{1 / 2}$ when $n=1 / 2$, and fit this equation and Eq. (8) to computational results for $E_{\mathrm{DHA}}$ versus $r-r_{1 / 2}$. In the fitting, we recall here that since a symmetrized expression is used, the average of the energy constants $E_{i i}$ of the two bonds $E_{i i}$ appears in Eq. (8) of Ref. 28. Unless careful precautions are taken, such as using Eq. (25), erroneous values for bond order results can be obtained and invalidate the comparison of computations with Eq. (8). We also note that the presence of work terms must be allowed for in separating computationally the two contributions $w^{r}$ and $E_{\mathrm{DHA}}$ in an energy profile versus bond length differences.

A key approximation is the separation of the free energy barrier into the sum of three contributions in Eq. (22). For further insight it would be useful to have a comparison of the KIE experimental data for reactions in solution (in which the second-order rate constants has been converted to a first order by a collision complex formalism) and the KIE for firstorder rate constant ( $k_{\text {cat }}$ in the usual enzyme terminology) for the reaction in the enzyme complex. The KIE affects $\Delta E_{\mathrm{DHA}}^{\circ}$, and perhaps $w^{r}$, depending on the origin of the latter. The $\mathrm{KIE}$ is expected to be similar for reactions in enzyme and the corresponding reaction in solution if the main difference is in $\Delta G_{\mathrm{prot}}^{*}(R)$. A similarity of the KIE results would provide evidence for separation of $\Delta G_{\text {prot }}^{*}(R)$ from the other two contributions, since $\Delta G_{\text {prot }}^{*}(R)$ is very different for the two systems.

Nuclear tunneling, not included in Eqs. (10), (18), and (22), is discussed in Sec. V.

\section{CHARGE TRANSFER SPECTRUM AND $\Delta E_{\text {total }}$}

In principle, a charge transfer (CT) spectrum is possible, as seen from Fig. 1. However, if the D-A distance has to become significantly smaller in order to facilitate a charge transfer, then the intensity of this CT absorption spectrum will be small, and indeed such a spectrum does not appear to have been observed. A vertical transition to the upper curve in Fig. 1 with its sharply repulsive nature, may spread out the intensity, while a nonvertical transition may have small intensity because of a poor overlap of the $\mathrm{H}$ wave function.

The isotopic results on pressure effects discussed in Ref. 31 suggest that pressure tends to convert $\beta$ to $\alpha$ paths in the

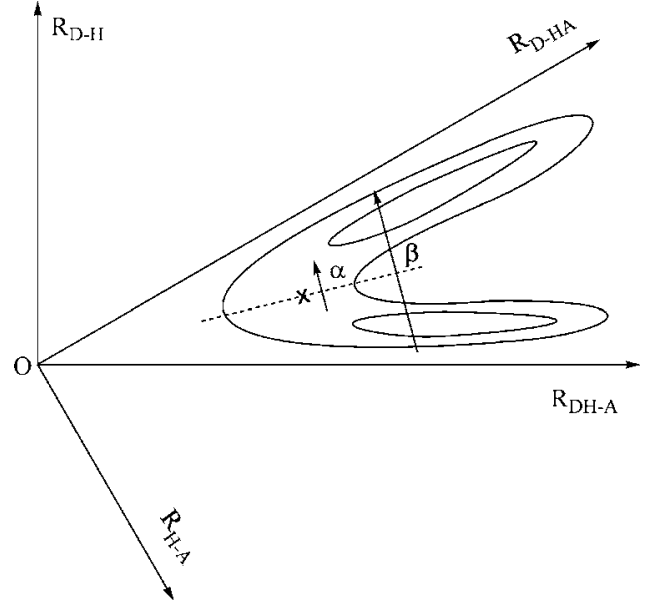

FIG. 2. Schematic potential energy surface for the reaction $A H+B \rightarrow A$ $+\mathrm{HB}$, using mass-weighted coordinates. $X$ denotes the saddle point and the $\alpha$ and $\beta$ paths are indicated. Polar coordinates $(R, \theta)$ are introduced, here and in Fig. 4, to define points and paths (cf. Ref. 31).

Fig. 2 given later. It would increase the intensity of any CT spectrum. High pressures have been used for studying other properties of biological systems, as in Ref. 32 and it would be interesting to see if such techniques could lead to the observation of charge transfer spectra in enzymes, or if the intensity is still too small.

The protein/solvent contribution to a CT spectrum (vertical for these heavier mass coordinates) is obtained by setting $m=0$ in Eq. (20a).

$$
h \nu_{\text {prot }}=\lambda_{o}+\Delta G_{\mathrm{prot}}^{\circ} .
$$

More generally, we consider a reaction coordinate commonly used in EVB computations $\Delta E_{\text {total }}$. In this case, the DHA and protein contributions to the free energy have the same functional form, approximately quadratic, and then they can be transformed such that a simple single reaction coordinate leads to the TS with a quadratic dependence of the free energy on that $\Delta E$ coordinate.

For example, if one energy "surface" of the reactants has the form $\frac{1}{2}\left(k_{a} q^{2}+k_{b} Q^{2}\right)$ and products' surface is displaced from it but with the same force constants, $\frac{1}{2}\left[k_{a}(q-a)^{2}\right.$ $\left.+k_{b}(Q-b)^{2}+\Delta\right]$, then the energy difference $\Delta E_{\text {total }}^{*}$ is $k_{a} q_{a}$ $+k_{b} Q_{b}+$ const. A new coordinate perpendicular to this plane in $(q, Q)$ space can be chosen, leading from the bottom of reactants' potential energy well to the plane where $\Delta E_{\text {total }}$ $=0$ (the TS) and then to the well of the products. Along this path, the energy for a system lying on the plane in $(q, Q)$ coordinate space for any given $\Delta E$ can be shown to be a quadratic function of $\Delta E$. Similar remarks apply when $k_{a} q_{a}^{2} / 2$ and $k_{a}(q-a)^{2} / 2$ describe free energy curves for the protein reorganization instead of one-dimensional potential energy curves.

However, when the two lowest adiabatic states are used instead of EVB states, the $G_{\text {prot }}$ and $E_{\text {DHA }}$ have different functional forms, a quadratic form for $G_{\text {prot }}$ and a ln cosh form for $E_{\mathrm{DHA}}$, given by Eqs. (12) and (8), respectively. For this reason, we employ a $\Delta E_{\text {total }}$ that depends on the two coordinates $m$ and $n$, rather than only one. We consider, thereby, the energy difference of the two lowest adiabatic 
electronic states, using Eq. (8). We assume that the sum of the potential energies for the two lowest adiabatic states is a constant or at most a linear function of $n$. (The motivation is the behavior of a simple two-state adiabatic approximation, in which the sum of the roots has a very simple form). The model would be inadequate when more than two adiabatic states are involved. Thus, from Eq. (8), the $E_{\mathrm{DHA}}$ of the upper adiabatic state contains a term $+\left(\lambda_{i} / 4 \ln 2\right)[2 \ln n+(1-n)$ $\ln (1-n)]$. A consequence is that the vertical energy difference of the DHA pair at any $n, \Delta E_{\mathrm{DHA}}(n, R)$, is

$$
\begin{aligned}
\Delta E_{\mathrm{DHA}}(n, R)= & \left(\frac{\lambda_{i}}{2 \ln 2}\right)[n \ln n+(1-n) \ln (1-n)] \\
& +n \Delta E_{\mathrm{DHA}}(R)-(1-n) \Delta E_{\mathrm{DHA}}^{p}(R),
\end{aligned}
$$

where $\Delta E_{\mathrm{DHA}}(R)$ and $\Delta E_{\mathrm{DHA}}^{p}(R)$ denote the vertical excitation energies of the reactants $(n=0)$ and of the products $(n=1)$ at $R$, respectively. There is also the vertical change in $w$, which for the present we write as $\Delta w$. The energy coordinate $\Delta E_{\text {total }}(n, m, R)$ is then given by

$$
\Delta E_{\text {total }}(n, m, R)=\Delta E_{\text {prot }}(m)+\Delta E_{\mathrm{DHA}}(n, R)+\Delta w,
$$

with $\Delta E_{\text {prot }}(m)$ given by Eq. $(20)$ and $\Delta E_{\mathrm{DHA}}(n, R)$ given by Eq. (28). For $\Delta w$, one might assume it to be a linear function of $m, \Delta w(m, R)=m \Delta w^{p}+(1-m) \Delta w^{r}$, where $\Delta w^{r}$ is the vertical change in $w^{r}$ for the reactants at $R$ and $\Delta w^{p}$ is that for the products at $R$.

\section{NUCLEAR TUNNELING}

The role of nuclear tunneling has been discussed in numerous articles, for example, in Philos. Trans. R. Soc. London, Ser. B. 361 (2006) and Chem. Rev. 106 (2006). The main focus of the present article until now has been on the nontunneling aspects of the reaction to see if some relatively simple expression can serve as a basis for characterizing the reactions and comparing with the experimental data and with computations. Such an analysis is facilitated if the tunneling factor is minimized by using $\mathrm{D}$ instead of $\mathrm{H}$, although the isotope effect plays an important role in understanding the reaction step. We treat the tunneling in this section, with the main aim of obtaining approximate simple expressions. For detailed numerical comparison with the experimental data current elaborate computational results can be of course invaluable.

For a series of related reactants nuclear tunneling is a maximum when $\Delta G^{\circ}=0$, as in examples in Refs. 33 and 34 and suggested in Ref. 35, i.e., it is a maximum in the symmetric case, for then the tunneling reaction coordinate through the TS is the $\mathrm{H}$ coordinate. In Fig. 2 the $\mathrm{H}$ coordinate would be along a polar coordinate $\theta$ or a chord. For this symmetric case, a relatively simple and accurate equation for the tunneling probability $\boldsymbol{P}(E)$ is given by Babamov and Marcus. ${ }^{36}$ The $\mathrm{H}$ tunneling will be clearly larger for Fig. 2 than for Fig. 3, since in the latter case the reaction coordinate at the TS has little or no contribution from H. Suitable analysis of tunneling computations from $\mathrm{H}$ transfers in enzymes or in solution can yield a functional form for the tunneling probability as a function of $|n-(1 / 2)|$.

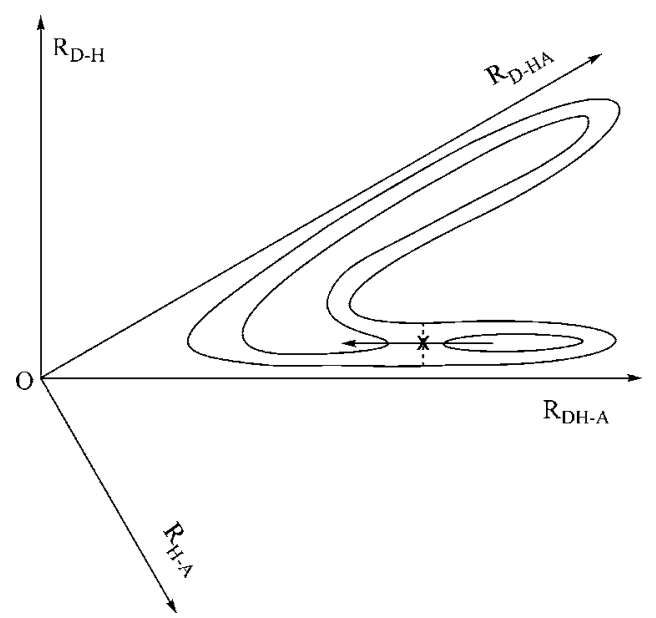

FIG. 3. Diagram illustrating the scenario where the saddle-point $X$ is reactants' well, the case of a very downhill reaction $\left(\Delta G_{R}^{\circ} \ll 0\right)$ (cf. Ref. 31).

Several tunneling cases have been described in Ref. 31, leading to figures such as Figs. 2 and 3, depending on the position of the transition state along the reaction coordinate. In these figures, the many-dimensional potential energy surface of the system is projected onto a two-dimensional subspace spanned by the two distances DH and HA. Figure 2 represents a reaction for which $\Delta E_{\mathrm{DHA}}^{\circ}(R) \sim 0$, the TS region occurring on a dotted line passing through $X$. In Fig. 3 the reaction is so downhill $\left(\Delta E_{\mathrm{DHA}}^{\circ}(R) \ll 0\right)$ that the TS region, again denoted by a dotted line passing through $X$, occurs in the entrance channel. The shaded regions in Figs. 4 and 5 denote the classically allowed regions for the systems in Figs. 2 and 3, respectively, for some given initial and final $\mathrm{H}$-quantum states and total energy $E$.

In a $\mathrm{H}$ transfer between two heavy atoms there is a continuum of reaction paths for proceeding from the well in the potential energy surface for the reactants $(\mathrm{DH}, \mathrm{A})$ to the well for the products (D, HA), paths such as $\alpha$ and $\beta$ in Fig. 2. This figure is adapted from one that appeared earlier. ${ }^{37}$ The

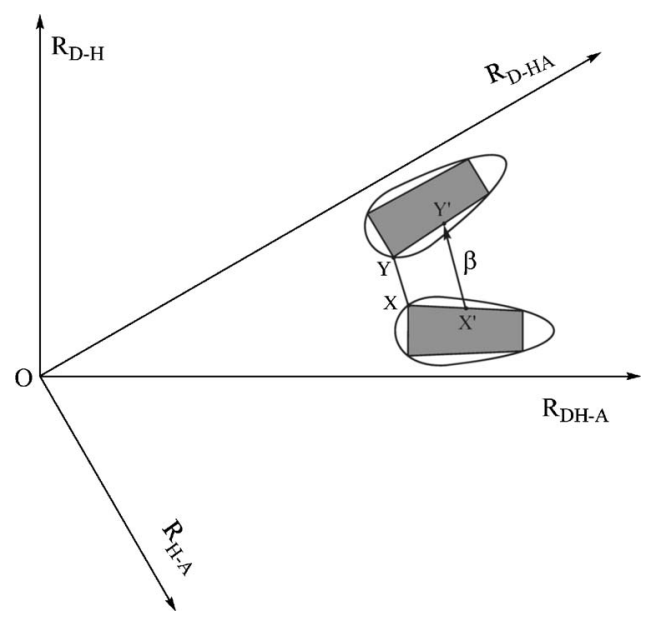

FIG. 4. Diagram showing the space swept out by a classical mechanical trajectory in reactants' well and by one in products' well and showing tunneling on a $\beta$ path from point $X^{\prime}$ on the boundary of reactants' distorted rectangle to point $Y^{\prime}$ on the boundary of products' distorted rectangle, including the nearest points $X^{\prime}=X$ and $Y^{\prime}=Y$ at the corners of the distorted rectangles (cf. Ref. 31). 


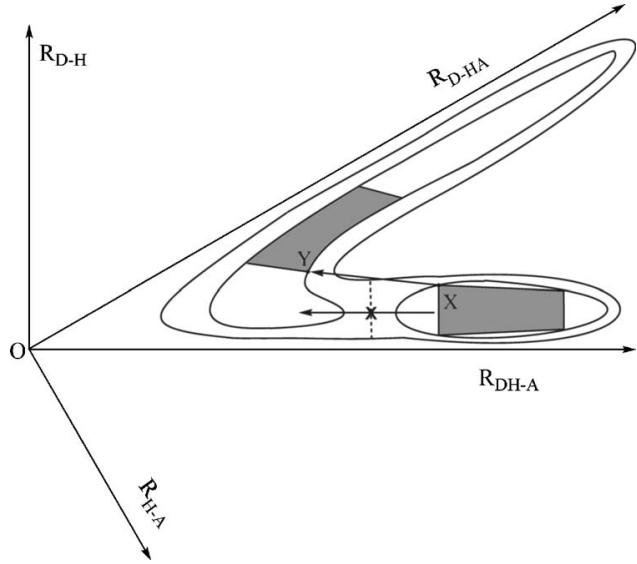

FIG. 5. Diagram similar to Fig. 4 but for the very downhill reaction in Fig. 3.

coordinates in the diagram are the usual mass-weighted coordinates in reaction rate theory, as in Ref. 38. The low mass of the $\mathrm{H}$ is reflected in the rather acute nature of the angle in Figs. 2-5. The paths there going from the well of the reactants, $\mathrm{DH}+\mathrm{A}$, to the well of the products, $\mathrm{D}+\mathrm{HA}$, range from those passing near the saddle-point $X$ (paths $\alpha$ ) to those occurring at longer DA distances (paths $\beta$ ). The $\beta$ paths typically involve tunneling of the $\mathrm{H}$, and there is a gradation between $\alpha$-and $\beta$-type paths, while $\alpha$ is a classically allowed path. A detailed discussion is given in Ref. 31 .

For the given H-quantum state and the given energy $E$ the wave function decays exponentially outside the distorted rectangles in Figs. 4 and 5. In wave mechanical terms the boundary of the distorted rectangle forms the "caustic." The system tunnels in Fig. 4 from any point $X$ or $X^{\prime}$ on the side of the distorted rectangle nearest products' well to a corresponding point on $Y$ or $Y^{\prime}$ on the nearest side of the distorted rectangle in products' well. The tunneling analysis can be made coordinate independent by choosing the path from $X^{\prime}$ to $Y^{\prime}$ variationally, instead of along an arc or along a chord. Various features of this tunneling are described in articles such as by Marcus and Coltrin ${ }^{39}$ and Fernandez-Ramos and $\operatorname{Truhlar}^{40}$ for the small curvature approximation, and by Ovchinnikova, ${ }^{41}$ Babamov and Marcus, ${ }^{36}$ and FernandezRamos and Truhlar ${ }^{42,43}$ for the large curvature approximation, and in the many articles cited therein. In recent years there have been many elaborations of the original concepts.

The rate constant can be written approximately as

$$
k_{\text {rate }} \cong(k T / h) \int \boldsymbol{P}(E) e^{-E / k T} d(E / k T) / Q
$$

where $Q$ is the partition function for the $R$ motion (the heavy atom motion) and $P(E)$ is the probability of reaction at energy $E$, as in Ref. 36. Classically, $Q=k T / h \nu$, where $\nu$ is the vibration frequency for the heavy atom motion in a well. For the symmetric case, $\Delta E_{\mathrm{DHA}}^{\circ}(R)=0$, and $P(E)$ for a $0 \rightarrow 0$ transition of the $\mathrm{H}$ state in a triatomic system is given by $\mathrm{Ba}-$ bamov and Marcus. ${ }^{36}$

$$
\boldsymbol{P}_{o o}(E)=\sin ^{2}\left(\xi_{o}^{s}-\xi_{o}^{a}\right),
$$

where the $\xi$ 's denote certain phase integrals. Higher transitions of the $\mathrm{H}$-quantum state $n \rightarrow n$ are easily included in $P(E)$. In that article only a two-coordinate system was treated and tested by comparison with accurate quantum mechanical results. The result can be extended approximately to more complex systems by writing

$$
\boldsymbol{P}_{o o}(E)=\exp \left(-\Delta G^{*} / k T\right) \sin ^{2}\left(\xi_{o}^{s}-\xi_{o}^{a}\right) .
$$

$E$ is measured from the lowest H-quantum state in the well.

When a semiclassical approximation is introduced in the tunneling regime the $\sin ^{2}\left(\xi_{0}^{s}-\xi_{0}^{a}\right)$ expression can be simplified but is now only applicable to the tunneling regime. We thus have from Ref. 36,

$$
\xi_{o}^{s}-\xi_{o}^{a}=\int_{-\infty}^{\infty} \nu_{\mathrm{H}}(R) e^{-K(R)} d t,
$$

where $\nu_{\mathrm{H}}(R)$ is the vibration frequency for the $\mathrm{H}$ motion in either well at any $R$, and $K(R)$ is the tunneling phase integral, $\int_{\theta_{1}}^{\theta_{2}}\left|p_{\theta}(R)\right| d \theta / \hbar$, at any $R$ from a point $X^{\prime}$ to $Y^{\prime}$ in Fig. 4 . It is written as $\theta=\theta_{1}$ to $\theta=\theta_{2}, \theta$ being the polar angle variable in that figure. When $K(R)$ decreases exponentially with increasing $R$ the integral, Eq. (31), is immediately integrated analytically, as in Ref. 36: $K(R)$ is expanded about $R=R_{o}$, the turning point for the classical $R$ motion, $K(R) \cong K\left(R_{o}\right)$ $+\left(R-R_{o}\right) K^{\prime}\left(R_{o}\right)$, where the prime denotes the $d K(R) / d R$ at $R=R_{o}$. The equation of motion $\mu \dot{R}^{2} / 2+U(R)=E$ is integrated in the vicinity of $R_{o}$, now defining $t=0$ to occur at $R=R_{o}$ and so yielding $R-R_{o}=\left(-U^{\prime} / \mu\right) t^{2}$. Upon writing $\nu_{\mathrm{H}}(R) \cong \nu_{\mathrm{H}}\left(R_{o}\right)$ and writing the latter as $\nu_{\mathrm{H}}$, we have

$$
\xi_{o}^{s}-\xi_{o}^{a}=\nu_{\mathrm{H}} e^{-K\left(R_{o}\right)} \int_{-\infty}^{\infty} e^{-K^{\prime}\left(-U^{\prime} / \mu\right) t^{2}} d t
$$

and upon evaluation of this integral we obtain

$$
\boldsymbol{P}_{o o}(E)=-\frac{2 \pi \mu \nu_{\mathrm{H}}^{2}}{U^{\prime} K^{\prime}} e^{-2 K\left(R_{o}\right)} e^{-\Delta G^{*} / k T} .
$$

Usually the preexponential factor in Eq. (35) is neglected, e.g., as in a related type of expression in Ref. 39, resulting there in a small displacement of their $\log P(E)$ versus $E$ plot from accurate quantum mechanical calculations over five decades. Equation (35) applies to the nuclear tunneling regime, whereas Eq. (32) applies whether or not there is nuclear tunneling.

If we use $\kappa$ to denote the ratio of tunneling to notunneling rate constants $\kappa=k_{\text {rate }}^{\text {tunn }} / k_{\text {rate }}^{\text {no tunn }}$, then one expects $\kappa=1$ when the TS is solely in the reactant channel or when it is solely in products' channel, and to be a maximum at $\Delta G^{\circ}=0$. A possible interpolative expression for experimental and computational tests is

$$
\kappa=\left(\kappa_{\max }\right)^{4 n(1-n)},
$$

so that when $n=1 / 2$, we have the symmetric case and $\kappa$ $=\kappa_{\max }$. On the other hand $\kappa=1$ when $n=0$ or $1, n$ being the value in the TS. An approximate version of Eq. (36) is

$$
\kappa \cong\left(\kappa_{\max }\right)^{1-\left(\Delta E_{\mathrm{DHA}}^{\circ} / \lambda_{i}\right)^{2}} .
$$


Equations (32)-(35) apply to the symmetric case $\left(\Delta E_{\mathrm{DHA}}^{\circ}(R)=0\right)$ and so give the value where the tunneling is a maximum and where the kinetic isotope effect $k_{\mathrm{H}} / k_{\mathrm{D}}$ is also a maximum. Not only is the $\mathrm{H}$ tunneling a maximum when $n=1 / 2$, but also the participation of the $\mathrm{H}$ motion in the reaction coordinate is greatest when $n=1 / 2$. Thus, a first approximation to test is

$$
k_{\mathrm{H}} / k_{\mathrm{D}} \cong\left(k_{\mathrm{H}} / k_{\mathrm{D}}\right)_{\max }^{4 n(1-n)}
$$

Thereby, we have $k_{\mathrm{H}} / k_{\mathrm{D}} \cong 1$ when the TS is solely in reactants' $(n=0)$ or in products' $(n=1)$ channel in $n$ space, as expected. The $n$ is again the value given earlier that maximizes the barrier in Eq. (8).

On increasing the asymmetry one obtains Fig. 3, an extreme case of an asymmetrical system. The deuterium KIE has decreased and the ${ }^{12} \mathrm{C} /{ }^{13} \mathrm{C}$ KIE, in contrast, increases: In an asymmetrical system $R$ becomes a more dominant contributor to the reaction coordinate and so the ${ }^{13} \mathrm{C}$ KIE increases while the deuterium KIE decreases, a result compared in Ref. 31 with the experimental data. Whereas the $\mathrm{H} / \mathrm{D}$ KIE is expected to be a maximum at $n=1 / 2$ and a minimum at $n=0$ and at $n=1$, the ${ }^{12} \mathrm{C} /{ }^{13} \mathrm{C}$ is expected to be a minimum at $n=1 / 2$.

This minimum of $k^{12} / k^{13}$ at $n=1 / 2$ arises for both nontunneling and tunneling reasons. At $n=1 / 2$ the reaction coordinate is largely the $\mathrm{H}$ motion and so the difference between the ${ }^{12} \mathrm{C}$ and ${ }^{13} \mathrm{C}$ contributions to the reduced mass for this motion is least, both classically and in any $\mathrm{C}$ nuclear tunneling component. When $n \neq 1 / 2$, the reaction coordinate now contains some component from the $\mathrm{C}$ and thus there are both classical and tunneling contributions by the $\mathrm{C}$, and so a larger C-KIE.

A possible functional form for the ${ }^{13} \mathrm{C}$ KIE is given by

$$
k_{12} / k_{13}=\left(k_{12} / k_{13}\right)_{\max }^{(1-2 n)^{2} f},
$$

where $n$ is the $n$ in the TS and $f=1$ or $n(1-n)$ depending on whether the ${ }^{13} \mathrm{C}$ KIE is a minimum only at $n=1 / 2$ or at $n$ $=0,1 / 2$ and 1 .

\section{CONCLUDING REMARKS}

A simple phenomenological expression has been obtained for the rate of $\mathrm{H}^{+}, \mathrm{H}^{-}$, and $\mathrm{H}^{\bullet}$ transfers for the nontunneling case in terms of several properties that can be used to characterize the reacting system, just as analogous properties have been used to characterize electron transfer systems. Such an analysis would be based both on experimental and computational results. The result is extended to include tunneling for the case of $\Delta E_{\mathrm{DHA}}^{\circ}(R)=0$. This tunneling result can be extended to finite $\Delta E_{\mathrm{DHA}}(R)$ when a functional form for the dependence of the tunneling probability/maximum tunneling probability on $\Delta E_{\mathrm{DHA}}^{\circ}(R)$ or on $|n-1 / 2|$ is assigned.

\section{ACKNOWLEDGMENTS}

It is a pleasure to acknowledge the support of this research by the Office of Naval Research and the National
Science Foundation and to acknowledge helpful discussions with Arieh Warshel, Amnon Kohen, Sharon HammesSchiffer, Don Truhlar, Jiali Gao, Judith Klinman, and Maria Michel-Beyerle.

${ }^{1}$ J. Gao, S. Ma, D. J. Major, K. Nam, J. Pu, and D. G. Truhlar, Chem. Rev. (Washington, D.C.) 106, 3119 (2006).

${ }^{2}$ A. Warshel, P. K. Sharma, M. Kato, Y. Xiang, H. Liu, and M. H. M. Olsson, Chem. Rev. (Washington, D.C.) 106, 3210 (2006).

${ }^{3}$ J. N. Gehlen, D. Chandler, H. J. Kim, and J. T. Hynes, J. Phys. Chem. 96, 1748 (1992).

${ }^{4}$ R. A. Marcus and N. Sutin, Biochim. Biophys. Acta 811, 265 (1985).

${ }^{5}$ J. Aqvist and A. Warshel, Chem. Rev. (Washington, D.C.) 93, 2523 (1993).

${ }^{6}$ S. R. Billeter, S. P. Webb, P. K. Agarwal, T. Iordanov, and S. HammesSchiffer, J. Am. Chem. Soc. 123, 11262 (2001).

${ }^{7}$ R. A. Marcus, Discuss. Faraday Soc. 29, 21 (1960).

${ }^{8}$ G. King and A. Warshel, J. Chem. Phys. 93, 8682 (1990).

${ }^{9}$ A. Warshel and R. M. Weiss, J. Am. Chem. Soc. 102, 6218 (1980).

${ }^{10}$ M. J. Gillan, J. Phys. C 20, 3621 (1987).

${ }^{11}$ G. A. Voth, D. Chandler, and W. H. Miller, J. Chem. Phys. 91, 7749 (1989).

${ }^{12}$ A. Warshel and Z. T. Chu, J. Chem. Phys. 93, 4003 (1990).

${ }^{13}$ W. J. Albery, Faraday Discuss. Chem. Soc. 74, 245 (1982).

${ }^{14}$ D. Borgis and J. T. Hynes, J. Phys. Chem. 100, 1118 (1996).

${ }^{15}$ D. Antoniou and S. D. Schwartz, Proc. Natl. Acad. Sci. U.S.A. 94, 12360 (1997).

${ }^{16}$ P. M. Kiefer and J. T. Hynes, J. Phys. Chem. A 106, 1834 (2002).

${ }^{17}$ P. M. Kiefer and J. T. Hynes, J. Phys. Chem. A 108, 11809 (2004).

${ }^{18}$ A. M. Kuznetsov and J. Ulstrup, Can. J. Chem. 77, 1085 (1999).

${ }^{19}$ R. A. Marcus, J. Chem. Phys. 43, 679 (1965).

${ }^{20}$ R. A. Marcus, J. Chem. Phys. 24, 966 (1956).

${ }^{21}$ A. A. Ovchinnikov and M. Y. Ovchinnikova, Zh. Eksp. Teor. Fiz. 56, 1278 (1969).

${ }^{22}$ Y. Georgievskii, C.-P. Hsu, and R. A. Marcus, J. Chem. Phys. 110, 5307(1999)

${ }^{23}$ N. R. Kestner, J. Logan, and J. Jortner, J. Phys. Chem. 78, 2148 (1974).

${ }^{24}$ A. Warshel, J. K. Hwang, and J. Aqvist, Faraday Discuss. 93, 225 (1992).

${ }^{25}$ R. A. Kuharski, J. S. Bader, D. Chandler, M. Sprik, M. L. Klein, and R. W. Impey, J. Chem. Phys. 89, 3248 (1988).

${ }^{26}$ C. N. Schutz and A. Warshel, J. Phys. Chem. B 108, 2066 (2004).

${ }^{27}$ H. S. Johnston, Adv. Chem. Phys. 3, 137 (1960).

${ }^{28}$ R. A. Marcus, J. Phys. Chem. 72, 891 (1968).

${ }^{29}$ L. Pauling, J. Am. Chem. Soc. 69, 542 (1947).

${ }^{30}$ C. Parr and H. S. Johnston, J. Am. Chem. Soc. 85, 2544 (1963).

${ }^{31}$ R. A. Marcus, Philos. Trans. R. Soc. London, Ser. B 361, 1445 (2006).

${ }^{32}$ G. Weber and H. Drickamer, Q. Rev. Biophys. 16, 89 (1983).

${ }^{33}$ R. P. Bell, The Proton in Chemistry (Cornell University Press, Ithaca, 1973).

${ }^{34}$ R. P. Bell, The Tunnel Effect in Chemistry (Chapman and Hall, London, 1980).

${ }^{35}$ J. Rucker, Y. Cha, T. Jonsson, K. L. Grant, and J. P. Klinman, Biochemistry 31, 11489 (1992).

${ }^{36}$ V. K. Babamov and R. A. Marcus, J. Chem. Phys. 74, 1790 (1981).

${ }^{37}$ R. A. Marcus, in Proceedings of Third Symposium Electrode Processes 1979, edited by S. Bruckenstein, J. D. E. McIntyre, B. Miller, and E. Yeager (Electrochemical Society, Princeton, 1980).

${ }^{38}$ S. Glasstone, K. J. Laidler, and H. Eyring, The Theory of Rate Processes (McGraw-Hill, New York, 1941).

${ }^{39}$ R. A. Marcus and M. E. Coltrin, J. Chem. Phys. 67, 2609 (1977).

${ }^{40}$ A. Fernandez-Ramos, D. G. Truhlar, J. Corchado, and J. EspinosaGarcia, J. Phys. Chem. A 106, 4957 (2002).

${ }^{41}$ M. Y. Ovchinnikova, Chem. Phys. 36, 85 (1979).

${ }^{42}$ A. Fernandez-Ramos, D. G. Truhlar, J. C. Corchado, and J. EspinosaGarcia, J. Phys. Chem. A 106, 4957 (2002).

${ }^{43}$ A. Fernandez-Ramos and D. G. Truhlar, J. Chem. Theory Comput. 1, $1063(2005)$. 